\title{
Planned Time Point Name
}

National Cancer Institute

\section{Source}

National Cancer Institute. Planned Time Point Name. NCI Thesaurus. Code C82539.

The literal identifier of a planned point in time. 